\title{
EDITORIAL
}

\section{Contrast-associated acute kidney injury is a myth: No}

\author{
Steven D. Weisbord ${ }^{1,2^{*}}$ and Damien du Cheryon ${ }^{3}$
}

(C) 2017 Springer-Verlag GmbH Germany, part of Springer Nature, and ESICM (outside the USA)

\section{Introduction}

The intravascular administration of iodinated contrast media has been a recognized etiology of acute kidney injury (AKI) for decades [1]. Recent studies have questioned the causal association of iodinated contrast administration with acute impairment in kidney function [2-7]. Review of specific lines of past scientific inquiry on contrast administration and AKI and critical analysis of recent research questioning this association demonstrates that contrast-associated AKI (CA-AKI) is not a myth.

\section{Discussion}

\section{The effects of contrast on the kidney}

Past research on the physiologic effects of intravascular contrast in the kidney supports the nephrotoxicity of iodinated contrast (Fig. 1). In some animal models, the intravascular administration of iodinated contrast results in decreased renal blood flow to and a reduction in the partial pressure of oxygen of the outer renal medulla, a segment of the kidney that is particularly vulnerable to perturbations in oxygen supply [8,9]. This adverse hemodynamic effect of contrast was also observed in studies of healthy human subjects using blood oxygen level-dependent MRI, in which the intravascular administration of iodinated contrast reduced renal medullary blood flow. Contrast administration in animals has also been shown to increase the generation of oxygen free radicals, an effect that is associated with a decrease in glomerular filtration [8]. Finally, in vitro studies demonstrate that iodinated contrast has adverse effects on mitochondrial enzyme activity and membrane function

\footnotetext{
*Correspondence: weisbordsd@upmc.edu

${ }^{1}$ Renal Section and Center for Health Equity Research and Promotion, VA Pittsburgh Healthcare System, Mailstop 111F-U, University Drive, Pittsburgh, PA 15240, USA

Full author information is available at the end of the article
}

and contributes to apoptosis of renal tubular epithelial cells $[10,11]$. Although findings in animal models do not necessarily translate to humans and the aforementioned studies in animals have certain methodologic limitations, these and other studies provide a pathophysiologic basis for the nephrotoxicity of iodinated contrast.

\section{Volume and type of contrast agent}

Several studies have identified an association of a higher volume of iodinated contrast with increased risk of AKI [12]. While such analyses are confounded by the recognition that sicker patients with more complex clinical presentations (who are hence at higher baseline risk for AKI) may undergo procedures that require higher volumes of contrast, this 'dose-response' relationship between contrast volume and risk of renal injury supports the nephrotoxic potential of these agents. Furthermore, past studies that compared the effects of different contrast agents support their causal association with AKI [13]. The initial contrast media used in clinical practice were 'high osmolal' with osmolalities several fold greater than blood (i.e., $1500-2000 \mathrm{mOsm} / \mathrm{kg}$ ). Following the introduction of 'low-osmolal' contrast media (osmolality $\sim 600-850 \mathrm{mOsm} / \mathrm{kg}$ ), clinical trials and meta-analyses demonstrated lower risk for CA-AKI with these agents compared with 'high-osmolal' media [14]. A differential risk of AKI was also observed in certain more recent studies that demonstrated lower rates of CA-AKI following procedures that used iso-osmolal iodixanol compared with certain 'low-osmolal' agents [15]. While differences between iodixanol and 'low-osmolal' contrast regarding the risk for AKI were not shown in all prior trials, one would not expect to see any differences in the incidence of renal injury with these agents if they had no adverse effects on the kidneys.

\section{望




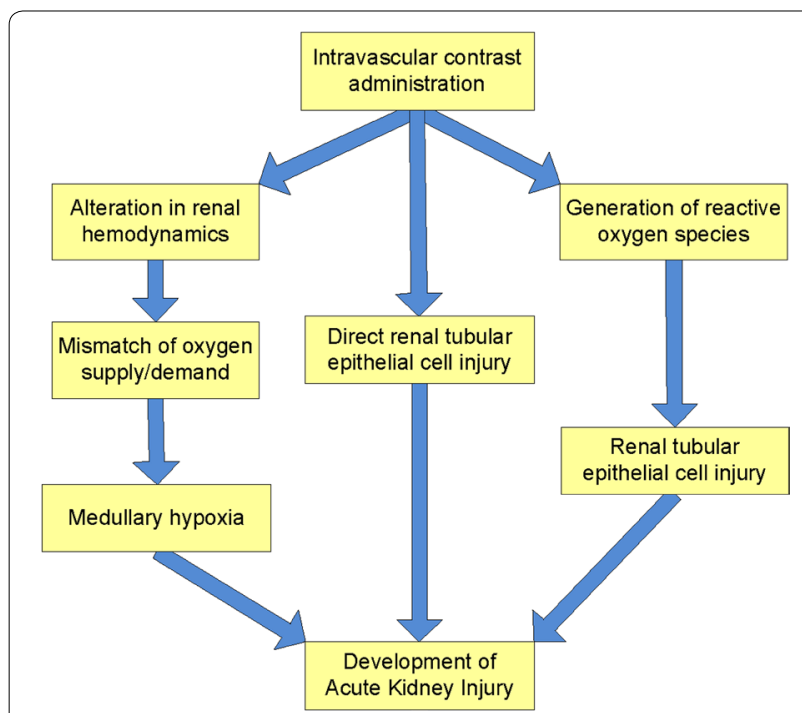

Fig. 1 Pathophysiology of acute kidney injury following intravascular contrast administration

\section{Recent studies questioning the existence of CA-AKI}

Notwithstanding past research that documented the pathophysiologic effects of iodinated contrast on the kidneys and differential risk of AKI based on volume and type of contrast agent, multiple recent studies have questioned the existence of CA-AKI [2-6]. A meta-analysis by McDonald and colleagues that included 13 studies with a total of 25,950 patients demonstrated that the risk of AKI following procedures with intravascular contrast administration was similar to the risk following procedures that did not utilize contrast (relative risk, 0.79; 95\% CI, 0.62-1.02) [3]. The authors also reported no differences in the need for dialysis or death based on the receipt of contrast. More recently, Wilhelm-Leen et al. compared the incidence of AKI in a large cohort of hospitalized patients who did and did not undergo contrast-enhanced procedures [7]. In adjusted analyses, the incidence of AKI was $5.1 \%$ in patients who received contrast compared with $5.6 \%$ in those who did not (adjusted odds ratio, 0.93; 95\% CI, 0.88-0.97).

While these and several other studies form the basis for the current hypothesis that CA-AKI does not exist, careful inspection of these studies demonstrates certain methodologic limitations that raise questions about the findings. First, all of these studies were retrospective observational analyses that relied on data that had been collected as part of routine clinical care. As such, the results were based solely on those patients in whom renal function was assessed prior to and following radiographic procedures. Furthermore, differential assessment of kidney function, regardless of reason, could not be fully accounted for in the analyses. Second, the use of preventive care to mitigate the risk of CA-AKI could not be fully evaluated. For example, demonstrating that the incidence of AKI is similar in patients who receive aggressive intravascular volume expansion before receiving iodinated contrast compared with patients who undergo non-contrast enhanced procedures would not establish that intravascular contrast is not nephrotoxic. Finally and most importantly, these studies could not fully account for factors that influenced providers' decisions regarding the use of intravascular contrast. Patients at higher baseline risk of AKI were almost certainly less likely to receive intravascular contrast than patients at low baseline risk. No degree of statistical adjustment or propensity score matching can fully account for all potential confounders or eliminate the effect of indication bias. In fact, the likelihood that there were substantial differences in baseline risks for AKI between patients who did and did not receive contrast is borne out in the findings of some of these studies that demonstrated statistically significantly lower rates of CA-AKI among patients who received contrast compared with patients who did not $[4$, 7]. Unless one believes that intravascular iodinated contrast is nephroprotective, this observation highlights the likelihood of confounding by indication in such studies.

\section{Conclusion}

In summary, prior research has elucidated pathophysiologic effects of iodinated contrast on the kidneys in animal models and humans, while studies documenting associations of volume and osmolality of contrast media with risk for renal injury support their nephrotoxic potential. Recent studies questioning the existence of CA-AKI have important methodologic limitations that confound interpretation of their findings. While secular trends including the use of lower volumes of less nephrotoxic contrast along with the widespread use of preventive care including intravascular volume expansion have likely contributed to decreased rates of CA-AKI and rendered severe renal injury a relatively rare complication of contrast administration alone, these factors have not eliminated the existence of this iatrogenic condition. Continued vigilance and appropriation of evidencebased preventive care in the highest risk patients remains essential.

\footnotetext{
Author details

${ }^{1}$ Renal Section and Center for Health Equity Research and Promotion, VA Pittsburgh Healthcare System, Mailstop $111 \mathrm{~F}-\mathrm{U}$, University Drive, Pittsburgh, PA 15240, USA. ${ }^{2}$ Renal-Electrolyte Division, Department of Medicine, University of Pittsburgh School of Medicine, Pittsburgh, PA, USA. ${ }^{3}$ CHU de Caen, Service de Réanimation Medicale, Caen, France.
} 


\section{Compliance with ethical standards}

\section{Conflicts of interest}

The authors declare that they have no competing interests. The opinions expressed in this article are those of the authors and do not necessarily represent those of the U.S. Department of Veterans Affairs.

Received: 20 November 2017 Accepted: 29 November 2017 Published online: 14 December 2017

\section{References}

1. Ansari Z, Baldwin DS (1976) Acute renal failure due to radio-contrast agents. Nephron 17:28-40

2. McDonald JS, McDonald RJ, Carter RE, Katzberg RW, Kallmes DF, Williamson EE (2014) Risk of intravenous contrast material-mediated acute kidney injury: a propensity score-matched study stratified by baselineestimated glomerular filtration rate. Radiology 271:65-73

3. McDonald JS, McDonald RJ, Comin J, Williamson EE, Katzberg RW, Murad MH, Kallmes DF (2013) Frequency of acute kidney injury following intravenous contrast medium administration: a systematic review and meta-analysis. Radiology 267:119-128

4. McDonald JS, McDonald RJ, Lieske JC, Carter RE, Katzberg RW, Williamson EE, Kallmes DF (2015) Risk of acute kidney injury, dialysis, and mortality in patients with chronic kidney disease after intravenous contrast material exposure. Mayo Clin Proc 90:1046-1053

5. McDonald JS, McDonald RJ, Williamson EE, Kallmes DF (2017) Is intravenous administration of iodixanol associated with increased risk of acute kidney injury, dialysis, or mortality? A propensity score-adjusted study. Radiology 285:414-424

6. McDonald JS, McDonald RJ, Williamson EE, Kallmes DF, Kashani K (2017) Post-contrast acute kidney injury in intensive care unit patients: a propensity score-adjusted study. Intensive Care Med 43:774-784
7. Wilhelm-Leen E, Montez-Rath ME, Chertow G (2017) Estimating the risk of radiocontrast-associated nephropathy. J Am Soc Nephrol 28:653-659

8. Bakris GL, Lass N, Gaber AO, Jones JD, Burnett JC Jr (1990) Radiocontrast medium-induced declines in renal function: a role for oxygen free radicals. Am J Physiol 258:F115-F120

9. Liss P, Nygren A, Revsbech NP, Ulfendahl HR (1997) Measurements of oxygen tension in the rat kidney after contrast media using an oxygen microelectrode with a guard cathode. Adv Exp Med Biol 411:569-576

10. Zager RA, Johnson AC, Hanson SY (2003) Radiographic contrast mediainduced tubular injury: evaluation of oxidant stress and plasma membrane integrity. Kidney Int 64:128-139

11. Hardiek K, Katholi RE, Ramkumar V, Deitrick C (2001) Proximal tubule cell response to radiographic contrast media. Am J Physiol Renal Physiol 280:F61-F70

12. Laskey WK, Jenkins C, Selzer F, Marroquin OC, Wilensky RL, Glaser R, Cohen HA, Holmes DR Jr (2007) Volume-to-creatinine clearance ratio: a pharmacokinetically based risk factor for prediction of early creatinine increase after percutaneous coronary intervention. J Am Coll Cardiol 50:584-590

13. Rudnick MR, Goldfarb S, Wexler L, Ludbrook PA, Murphy MJ, Halpern EF, Hill JA, Winniford M, Cohen MB, VanFossen DB (1995) Nephrotoxicity of ionic and nonionic contrast media in 1196 patients: a randomized trial. The lohexol Cooperative Study. Kidney Int 47:254-261

14. Barrett BJ, Carlisle EJ (1993) Metaanalysis of the relative nephrotoxicity of high- and low-osmolality iodinated contrast media. Radiology 188:171-178

15. Aspelin P, Aubry P, Fransson SG, Strasser R, Willenbrock R, Berg KJ (2003) Nephrotoxic effects in high-risk patients undergoing angiography. N Engl J Med 348:491-499 